\title{
Bimodal in vivo imaging provides early assessment of stem-cell-based photoreceptor engraftment
}

CRJ Laver' ${ }^{1}$, AL Metcalfe', L Szczygiel², A Yanai' ${ }^{1}$ MV Sarunic ${ }^{3}$ and K Gregory-Evans ${ }^{1}$

\begin{abstract}
Purpose Subretinal transplantation of stem-cell-derived photoreceptor precursor cells (PPCs) is a promising and innovative approach to treating a range of blinding diseases. However, common barriers to efficient preclinical transplantation comes in the form of suboptimal graft architecture, limited graft survival, and immune-rejection, each of which cannot be assessed using conventional in vivo imaging (ie, rodent ophthalmoscopy). With the majority of PPCs reported to die within the first few weeks after transplantation, understanding the mechanisms of graft failure, and ultimately devising preventative methods, currently relies on lengthy end point histology.
\end{abstract}

To address these limitations, we hypothesized that combining two imaging modalities, optical coherence tomography (OCT) and fluorescence confocal scanning laser ophthalmoscopy (fcSLO), could provide a more rapid and comprehensive view of PPC engraftment.

Methods Human ESC-derived PPCs were transplanted into 15 retinal dystrophic rats that underwent bimodal imaging at 0,8 , and 15 days posttransplant.

Results Bimodal imaging provided serial detection of graft: placement, architecture, and survival; each undetectable under ophthalmoscopy. Bimodal imaging determined graft placement to be either: subretinal $(n=7)$, choroidal $(n=4)$, or vitreal $(n=4)$ indicating neural retinal perforation.

Graft architecture was highly variable at the time of transplantation, with notable redistribution over time, while complete, or near complete, graft loss was observed in the majority of recipients after day 8 . Of particular importance was detection of vitreal aggregates overlying the graft-possibly an indicator of host-site inflammation and rejection.

Conclusion Early real-time feedback of engraftment has the potential to greatly increase efficiency of preclinical trials in cell-based retinal therapeutics.

Eye (2015) 29, 681-690; doi:10.1038/eye.2015.24; published online 13 March 2015

\section{Introduction}

An important stage in preclinical efforts to develop effective, photoreceptor-based therapeutics for blinding retinal diseases is transplantation to the subretinal space of small mammals. However, these trials can be highly inefficient. This is not only due to surgical complications and poor rates of engraftment but also to the inability of conventional imaging to detect these problems early after transplantation.

Rodent subretinal transplantation surgery poses challenges that are uncommon in larger animal models. Significant among these problems is the small diameter of the rodent eye (eg, 3-4 $\mathrm{mm}$ in the adult rat) and presence of a lens taking up $\sim 50-60 \%$ of posterior-segment volume $^{1,2}$ - characteristics that limit the feasibility of conventional clinical approaches to subretinal injection (ie, trans-vitreal entry to the subretinal space). Though seldom reported, these factors have led to high surgical-attrition rates that greatly reduce efficiency of longerterm functional studies. Some trials, for instance, suffer from 22 to $40 \%$ attrition due to surgical misplacement of the graft alone 3,4 -attrition rates that do not include other common sources of engraftment failure (eg, suboptimal graft architecture, survival, and rejection), each of
${ }^{1}$ Department of

Ophthalmology and Visual Sciences, Faculty of Medicine, University of British Columbia, Vancouver, BC, Canada

${ }^{2}$ Department of Molecular Biology and Biochemistry, Faculty of Science, Simon Fraser University, Burnaby, BC, Canada

${ }^{3}$ School of Engineering Science, Faculty of Applied Science, Simon Fraser University, Burnaby, BC, Canada

Correspondence: K Gregory-Evans, Department of Ophthalmology and Visual Science, Faculty of Medicine, University of British Columbia, 2550 Willow Street, Vancouver, BC V5Z 3N9, Canada Tel: +604 875 5529; Fax: +604 8755275 E-mail: kge30@mail.ubc.ca

Received: 26 August 2014 Accepted in revised form: 23 January 2015 Published online: 13 March 2015 
which cannot be detected by conventional in vivo imaging (ie, rodent ophthalmoscopy).

Although various functional assessments can be used to evaluate photoreceptor graft success, ${ }^{5-7}$ these can only be used in long-term studies once functional integration has been achieved. Remarkably, a handful of studies have shown some restoration of vision in photoreceptor precursor cell (PPC) recipients, ${ }^{6-9}$ though graft integration and survival remains too low to be clinically relevant. Short-term assessment in serial studies aimed at addressing these barriers currently relies on imaging, such as ophthalmoscopy and end point histology. Given the considerable time and resource costs associated with serial and end point analyses, early graft assessment is essential for efficient, large-scale preclinical trials in retinal cell-based therapeutics. In this study, we proposed, for the first time, to assess the value of bimodal in vivo imaging, combining optical coherence tomography (OCT) and fluorescence confocal scanning laser ophthalmoscopy (fcSLO), to achieve a more comprehensive and timely assessment of PPC engraftment.

\section{Materials and methods}

\section{Animals}

Animals were treated in accordance with the Canadian Council for Animal Care mandates, the ARVO Statement for the Use of Animals in Ophthalmic and Vision Research, and with experimental protocols approved by University of British Columbia Animal Care Committee. The rodent lines used, S334ter-4 and P23H rats, were generously provided by Dr Matthew LaVail, University of California, San Francisco, USA. These rodent models of retinitis pigmentosa express a mutated rhodopsin gene, leading to slow and fast retinal degeneration, respectively. ${ }^{10}$ Transplant recipients $(n=15)$, ranging from P30 to P70, underwent oral administration of the immunosuppressant, cyclosporine-A (Neoral, Novartis, East Hanover, NJ, USA) at $210 \mathrm{mg} / \mathrm{l}$, from a minimum of 2 days prior to transplantation until euthanasia.

\section{Transplantation}

Human ESC-derived PPCs (day 18 of directed differentiation) were produced, as per the protocol by Yanai et al, ${ }^{11}$ and labeled with intracellular fluorescent quantum dots, Qtracker 655 (Life Technologies, Carlsbad, CA, USA), QT, as per the manufacturer's specifications, $1 \mathrm{~h}$ before transplantation and then kept on ice. We chose QT for its reported long-term retention within target cells ${ }^{12}$ and its long-term photo-stability and low toxicity; ${ }^{13,14}$ the latter, a result of it being sequestered within intracellular vesicles. ${ }^{15}$ The following surgical procedure was done under a dual-headed ophthalmic surgical microscope (Zeiss, Oberkochen, Germany), which also served as our ophthalmoscope. Recipients were anesthetized with isoflurane, host-eye anesthetized with proparicaine (Alcon, Fort Worth, TX, USA) preceding insertion of lid-retractors, and pupils dilated with $1 \%$ tropicamide (Bausch and Lomb, Rochester, NY, USA). Povidone-iodine (Alcon) antimicrobial solution was used to wash the eye, lids, surrounding skin, and fur of the recipient. Tear-gel (Alcon) was applied to the cornea throughout the surgery. Filtersterilized 2\% Nile Blue (Sigma-Aldrich, St Louis, MO, USA) dye (in dPBS) was used to mark limbal-conjunctiva at the temporal and inferior midlines for orientation of the eye. Host conjunctiva was then reflected (one-fourth-onesixth circumference), followed by rotation of the globe by grasping the limbal-conjunctiva with micro-forceps to visualize the inferior-temporal sclera. A lancing incision was made with 31-guage beveled needle inserted tangentially through the sclera toward the posterior pole until the one-third of the bevel was in the subretinal space. PPCs (with Trypan Blue, Life Technologies, based on viable cell counts of $>95 \%$ ) were maintained on ice before delivery through this incision using a 34-gauge needle attached to a $10 \mu$ l Gastight Syringe (Hamilton, Reno, $\mathrm{NV}$, USA); the syringe was mounted in a stereotaxic micromanipulator to decrease trauma and increase injection reproducibility. Following graft injection ( 55 000-70 000 cells in $3 \mu \mathrm{l}$ of sterile dPBS), the needle was removed after $1 \mathrm{~min}$. After transplantation, fcSLO and OCT (methods detailed below) were used to assess the graft and host (transplantation and imaging is outlined in Figure 1a). Recipients were then allowed to recover in an incubator with host eyes treated with: proparicaine analgesic drops, tobramycin $(0.3 \%)$ ophthalmic antibiotic ointment, and tear gel (all from Alcon).

\section{Bimodal imaging (fCSLO and OCT)}

In vivo imaging was performed on recipients at: 0,8 , and 15 days posttransplant using our custom fCSLO and OCT machine. ${ }^{16,17}$ For fcSLO, a 532-nm frequency-doubled Nd: YAG laser was used for back-reflectance imaging and fluorescence excitation. Band pass optical filters (561$700 \mathrm{~nm}$ detection range) were used to visualize the fluorescence from the graft-label QT (emission maxima of $655 \mathrm{~nm}$ ). The presence and extent of aggregation and dispersion of the QT fluorescence were assessed. For OCT imaging, b-scans of the retina were used to assess both the graft and host. OCT data were processed using custom software. ${ }^{16}$ For both imaging modalities, the contralateral, non-injected eye, served as a negative control. 
a

b
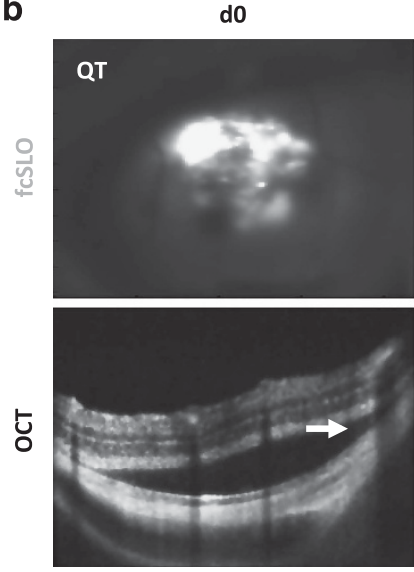

c


d8
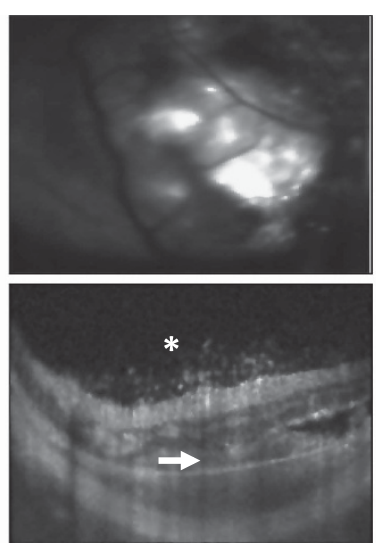

do
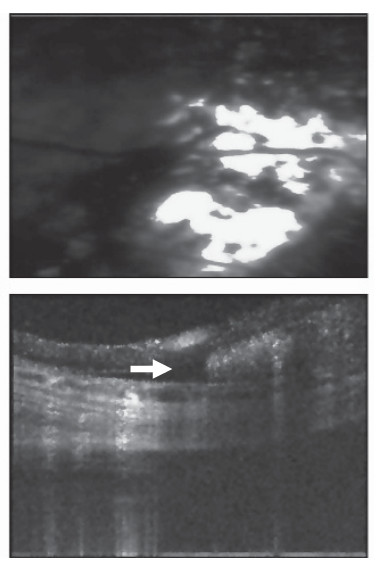

d15
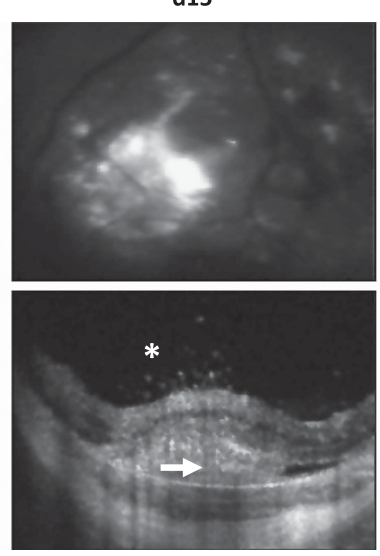

do


d15 - No transplant

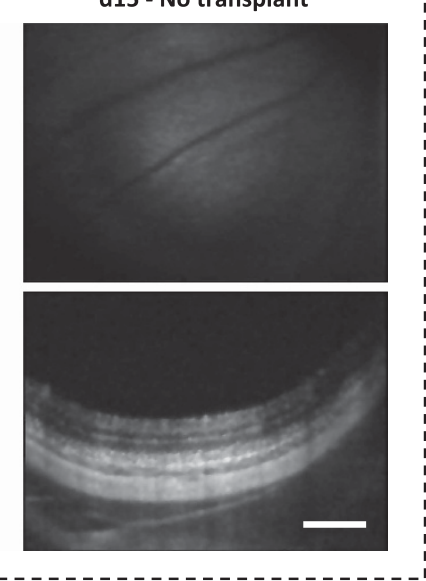

do
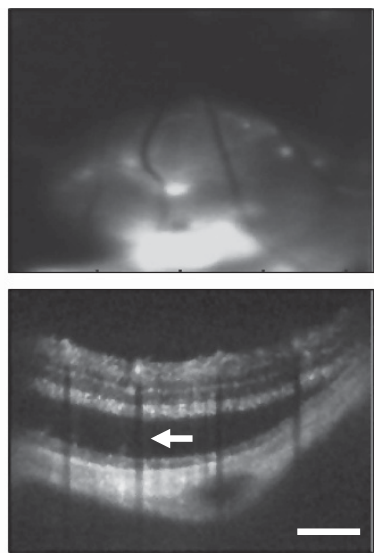

Figure 1 Transplantation and bimodal (OCT-fCSLO) in vivo imaging of subretinal PPC-grafts. (a) Overview of PPC transplantation via bolus injection into the subretinal space of retinal dystrophic rats. (b) Bimodal imaging was performed at each time point: day 0 (d0), day 8 (d8), and day 15 (d15); Qtracker (QT) PPC-graft label appears white on fcSLO images. OCT showing subretinal bleb formation and graft (arrowheads); note subretinal bleb contains host cells at d15. Vitreal aggregates atop graft site, an indicator of host-site inflammation (asterisks; also see Supplementary Video S1 of d15 recipient: 1$)$. Results are representative images $(n=4$, recipients: 1, 2, 7, and 11). Un-injected contralateral eyes served as a negative 'No transplant' control (dashed box). (c) Representative images of variable graft architecture (aggregation, dispersion, and thickness) at the time of transplantation (d0) ( $n=4$, recipients: 2, 3, 7, and 11). (b, c) Scale bars are $250 \mu \mathrm{m}$.

\section{Tissue processing}

Following euthanasia, recipient eyes were marked at the inferior midline and nasal equator with $2 \%$ Nile Blue dye for orientation in cryomolds and then enucleated and fixed in $10 \%$ buffered formalin for $24 \mathrm{~h}$. This was followed by cryopreservation treatments consisting of: two 10-min 
washes in PBS, dehydration in 20\% sucrose (in PBS) for 90 min, mounting in Peel-a-Way Embedding Molds (RR12 from ProSciTech, Thuringowa Central, Australia) containing Polyfreeze Tissue Freezing Medium (Polysciences, Warrington, PA, USA), and then freezing on dry-ice with subsequent storage at $-80^{\circ} \mathrm{C}$. Cryosections of $10-\mu \mathrm{m}$ thick were produced and stored at $-80^{\circ} \mathrm{C}$.

\section{Immunofluorescence}

Antibodies against the human-specific cell surface marker, Tra185, were used to qualify the presence and location of PPCs in transplant recipients and human cells in co-culture experiments. To do so, fixed tissue or cell cultures (that underwent the same fixation procedure as above, except for a duration of $20 \mathrm{~min}$ ) were washed in PBS and then blocked with 2\% normal goat serum (Vector Laboratories, Burlingame, CA, USA) in PBS for $1 \mathrm{~h}$, followed by washing with PBS. Endogenous biotin and streptavidin were blocked using the Streptavidin/ Biotin Blocking Kit (Vector Laboratories), as per the manufacturer's specifications. Biotinylated mouse antihuman Tra185 primary antibody (BAM3194 clone, R\&D Systems, Minneapolis, MN, USA) was applied to the tissue at a 1:100 dilution for 16h. Following washing with PBS, a streptavidin-conjugated Northern Lights (NL557, R\&D Systems) was applied at a 1:4000 dilution for $1 \mathrm{~h}$. Cell nuclei were labeled with Hoechst-33342 (Sigma-Aldrich) at $10 \mu \mathrm{g} / \mathrm{ml}$ in PBS for $5 \mathrm{~min}$ and followed by tissue mounting in Fluoromount-G (Southern Biotech, Birmingham, AL, USA). Tra185 antibody was validated using independent- and cocultures of human and rat cell lines: hESC-derived PPCs, retinal pigment epithelium (RPE) cell lines, rat RPE ${ }^{18}$ and human ARPE19, ${ }^{19}$ as well as on histological sections of PPC graft recipients at day 0 posttransplant. For non-antibody labeled histology, only the above nuclei labeling was applied.

\section{Confocal microscopy}

Microscopy of retinal sections was performed on either: a Zeiss LSM 510 META Confocal Laser-scanning System attached to a Zeiss Axiovert $200 \mathrm{M}$ inverted microscope, or a Zeiss Axioplan 2 microscope. QT or Tra185 signal was detected under epi-fluorescence illumination preceding acquisition of XY optical sections and Z-stacks. Image processing, including $3 \mathrm{D}$ rendering of images (18-22 images per 10- $\mu \mathrm{m}$ thick XYZ stack), was done using the ZEN 2012 software (Zeiss).

\section{Results \\ Detection of subretinal graft and indicators of host-site inflammation}

Bimodal in vivo imaging, combining OCT and fCSLO, serially detected the presence of subretinal graft and its location across the fundus (Figure $1 \mathrm{~b})(n=4$, recipients: 1, 2, 7, 11; see Table 1 for bimodal imaging observations for each recipient). For these four recipients, redistribution of the graft over time was particularly evident under fcSLO. Concurrently, OCT detected microscopic vitreal aggregates overlying the host site at 8 and 15 days posttransplant (Figure 1b, asterisks; $n=4$, recipients: 1, 2, $7,11)$. This feature could represent vitritis and local inflammation and thus a possible sign of graft rejection. Importantly, these vitreal aggregates were not detected in any subretinal transplant recipient at day 0 (Figures $1 \mathrm{~b}$ and c) nor found over areas of the retina that did not have graft underneath (eg, day 15 recipient: 1, see Supplementary Video S1). Ophthalmoscopy detected subretinal bleb formation on the day of transplantation, indicating placement in the correct ocular layer. However, it was unable to confirm graft presence, specific fundus location, or redistribution, as graft could not be resolved through the neural retina; microscopic host-site vitreal aggregates were also not detectable.

\section{In vivo assessment of graft architecture}

Bimodal imaging enabled assessment of subretinal graft architecture. This was markedly variable in aggregation, dispersion, and thickness immediately after transplantation within and among recipients (Figure 1c; $n=4$, recipients: $2,3,7$, and 11). Ophthalmoscopy could not detect these features.

\section{Detection of graft misplacement and trauma}

Bimodal imaging and ophthalmoscopy provided similar detection of graft-misplacement and common forms of surgical trauma.

In certain recipients, both a subretinal bleb and vitreal clouding atop the bleb was identified under ophthalmoscopy directly after transplantation, a clear indication of graft leaking into the vitreous through a fullthickness retinal tear caused by the injection needle. This graft clouding could also be visualized using bimodal imaging (Figure 2a, asterisks; $n=4$, recipients: 12-15). For these recipients, fcSLO reported a decrease in QT signal over time, indicating that the vitreal clouds were losing graft and therefore becoming predominantly composed of host material/cells (likely immune cell infiltrates, as no hemorrhaging was noted under 
Table 1 Bimodal in vivo imaging findings for PPC transplant recipients

\begin{tabular}{|c|c|c|c|c|c|c|c|}
\hline \multirow{3}{*}{$\begin{array}{l}\text { Recipient designation (1-15, grouped } \\
\text { by injection site outcome) }\end{array}$} & \multicolumn{3}{|c|}{ OCT findings } & \multicolumn{3}{|c|}{$f_{C} S L O$ findings } & \multirow{3}{*}{$\begin{array}{l}\text { Injection site } \\
\text { (noted complications) }\end{array}$} \\
\hline & \multicolumn{3}{|c|}{ Day } & \multicolumn{3}{|c|}{ Day } & \\
\hline & 0 & 8 & 15 & 0 & 8 & 15 & \\
\hline 1 & SB & SB, VA & SB, VA & + & + & + & Subretinal \\
\hline 2 & SB & $\mathrm{SB}, \mathrm{VA}$ & $\mathrm{SB}, \mathrm{VA}$ & + & + & + & Subretinal \\
\hline 7 & SB & SB, VA & SB, VA & + & + & + & Subretinal \\
\hline 11 & SB & SB, VA & SB, VA & + & + & - & Subretinal \\
\hline 10 & SB & SB & SB & + & - & - & Subretinal (GR) \\
\hline 3 & SB & SB & & + & + & & Subretinal \\
\hline 8 & SB & SB & a & + & a & a & Subretinal (Cat.) \\
\hline 4 & NAD & NAD & & + & - & & Choroidal \\
\hline 5 & NAD & VA & & + & - & & Choroidal \\
\hline 6 & NAD & a & & + & a & & Choroidal (Cat.) \\
\hline 9 & NAD & NAD & NAD & + & - & - & Choroidal \\
\hline 12 & $\mathrm{VC}$ & $\mathrm{VC}$ & $\mathrm{VC}$ & + & + & + & Vitreal (NRP) \\
\hline 13 & VC & VC & VC & + & + & - & Vitreal (NRP) \\
\hline 14 & $\mathrm{VC}$ & $\mathrm{VC}$ & $\mathrm{VC}$ & + & + & - & Vitreal (NRP) \\
\hline 15 & $\mathrm{VC}$ & NAD & VA & + & - & - & Vitreal (NRP) \\
\hline
\end{tabular}

Abbreviations: Cat., cataract; GR, graft reflux; NAD, nothing abnormal detected. Ophthalmoscopy observations were in concordance with OCT findings above. Ophthalmoscopy could only detect subretinal blebs (SBs) at day 0 and vitreal clouding (VC, graft in the vitreous resulting from neural retinal puncture, NRP), while microscopic vitreal aggregates (VAs, indicators of host-site inflammation) overlying the graft site could not be detected. NAD, Cat., and GR were observed during injection. Presence $(+)$ or absence $(-)$ of fluorescent graft label (Qtracker) across the fundus; heterogeneous signal distribution and redistribution was seen for all recipients with positive signal over time. Blank boxes indicate end point at previous time point. ${ }^{a}$ Cataract formation precluded complete bimodal imaging; OCT was able to resolve the retina and choroid at early stages of cataract formation; however, fcSLO could not penetrate the cataract.

ophthalmoscopy nor was blood-induced Rayleigh scattering present under OCT).

Another surgical complication encountered using the trans-scleral injection approach was transplantation to the incorrect ocular layer, most often (supra)choroidal, seen here and in other studies. ${ }^{20}$ Such events were clearly observed under OCT by the absence of a subretinal bleb at a host site where QT was observed under fcSLO (Figure $2 b ; n=3$, recipients: 4-6). Notably, ophthalmoscopy could not detect this form of misplacement, though graft injected into the choroid could also not be resolved from vasculature under OCTcombination of the two modalities (OCT and fcSLO) were required to determine this misplacement. Complete, or near complete, QT signal loss was also observed after day 8 or 15 in the majority of recipients ( $n=8$, recipients: 4,5 , 9-11, and 13-15), further illustrating bimodal imaging's ability to track graft loss (Figure 2c). Recipient number 8 (Table 1) could not undergo complete bimodal imaging owing to cataract formation by day 8 (at which time only OCT could penetrate the cataract).

\section{Concordance with end point histology}

We next assessed the concordance of bimodal imaging with end point histology. Our in vivo imaging accurately reflected recipient histology showing: graft placement in the subretinal space with variable graft architecture, both diffuse and large aggregates (Figure 3a), signs of graft loss (lack of human-cell marker, Tra185) at 15 days posttransplant (Figure $3 \mathrm{~b}$ ), but graft presence at day 0 (Figure 3c), while graft misplacement, such as injection into the choroid (Figure 3d), was also detected.

\section{Graft survival and QT location}

OCT detected both diffuse and large subretinal aggregates that, although spatially coinciding with QT under fcSLO at day 15 (Figure 1b), reflected cellular debris and infiltrating host cells when evaluated by end point histology (Figures 3a and b). We also observed that QT signal is lost during our IHC washing procedures (Figure $3 b$ ) and is independent of extended tissue-fixation (data not shown). However, PPC grafts labeled with QT are still detected using Tra185 antibodies after the QT signal is lost (day 0, Figure 3c).

With the aid of 3D rendering of optical Z-stacks, we were able to clearly detect QT within at least one host cell type-the RPE (Figure 3e). This resulted from either RPE uptake of lone QT or phagocytosis of PPC-graft containing QT. The latter scenario was observed in vitro here (Figure 3f) and in others studies ${ }^{21,22}$ and is expected given the RPE's phagocytic roles. ${ }^{23}$ 
a

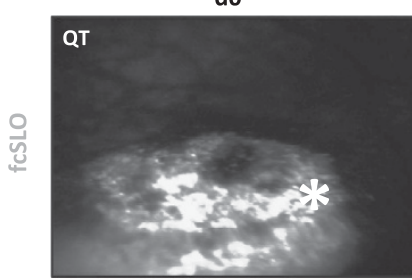

Ł

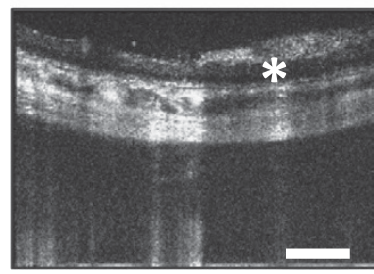

b
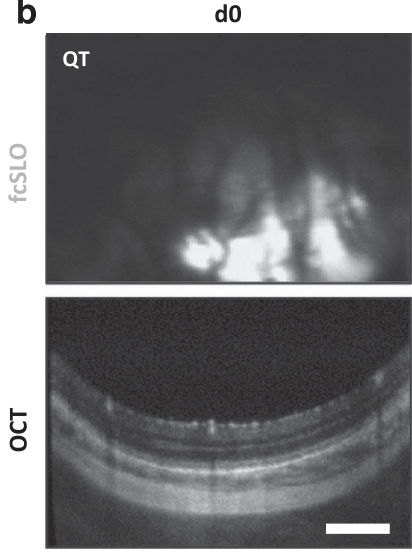

d8
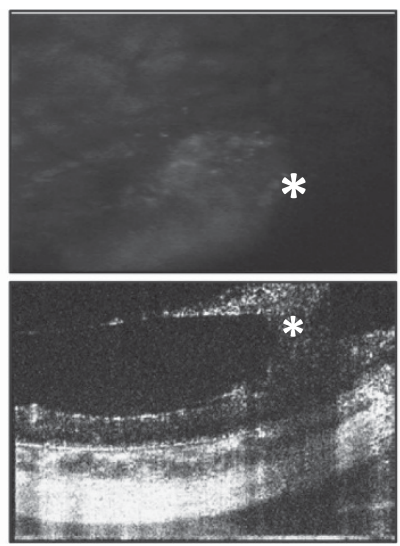

C
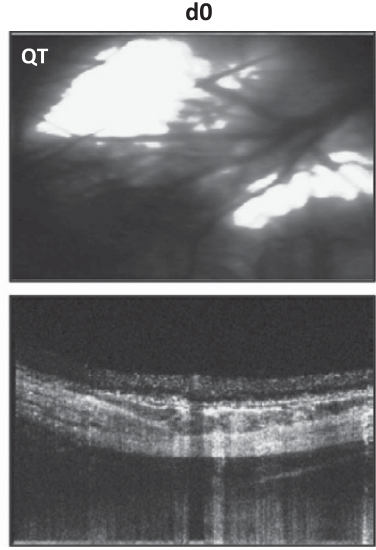

d15

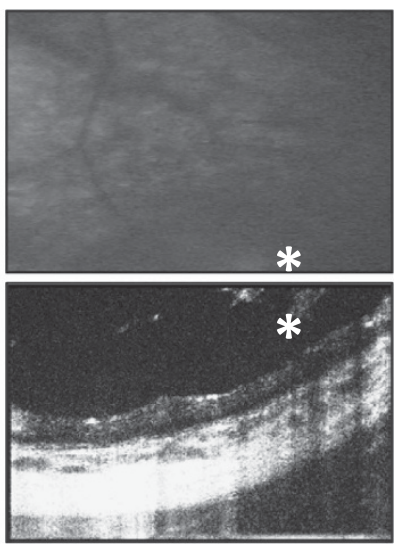

d8
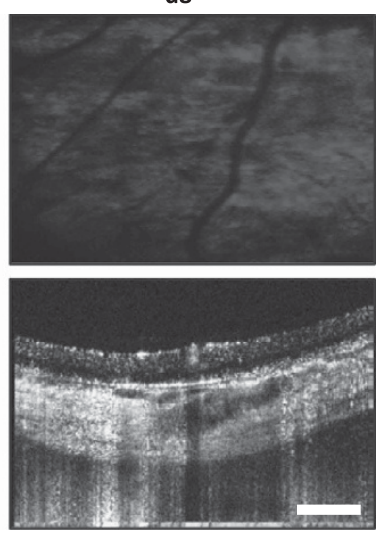

Figure 2 Detection of trauma, PPC-graft misplacement, and graft loss using bimodal (OCT-fcSLO) imaging. (a) Bimodal imaging showing epiretinal and vitreal graft clouding (asterisks) at day 0 (d0) indicating the presence of a full thickness retinal tear, while at day 8 (d8) and day 15 (d15) these aggregates are composed of mainly host cells; Qtracker (QT) loaded PPCs appear white on fcSLO images. Results are representative images $(n=4$, recipients: $12-15)$. (b) Misplacement of graft in the choroid ( $n=3$, recipients: 4-6). (c) QT signal loss indicating graft loss from host site after 8 or 15 days posttransplant $(n=8$, recipients: 4, 5, 9-11, and 13-15). (a-c) Scale bars are $250 \mu \mathrm{m}$.

\section{Discussion}

\section{Real-time detection of graft placement and survival}

Comprehensive in vivo assessment of subretinal grafts is essential for streamlining preclinical trials in cell-based retinal therapeutics. This will avoid prolonging experiments in animals where engrafted cells are incorrectly placed, under immune attack, or have died early in the experimental protocol. Although conventional in vivo imaging (rodent ophthalmoscopy) allows for some initial confirmation of subretinal bleb formation, $9,24,25$ implying transplantation to the correct retinal layer, it is ineffective at determining subretinal graft presence past the day of transplantation. This is because ophthalmoscopy views the retina en face, making blebs of smaller profile harder to identify; moreover, aside from host erythrocytes (in cases where subretinal hemorrhaging occurs), graft and host cells cannot be differentiated through the neural retinal. In addition to the obvious benefits of confirming graft placement before proceeding with costly downstream analyses, bimodal imaging can also detect other adverse events early on, such as microscopic indicators of host-site inflammation (ie, vitreal aggregate formation overlying the engraftment site) as well as graft loss (indicated by decreases in graft fluorescence). In future preclinical trials, this feature could help streamline immunosuppression regimes and enable more rapid feedback of various approaches for increasing graft survival-to date, one of the most challenging barriers to overcome in the development of a photoreceptor replacement therapy, as $>90 \%$ of even the most promising syngeneic-PPCs die within 2 weeks posttransplant. $^{9}$

\section{The importance of graft architecture}

Though graft placement and survival are critical to transplant efficacy, graft architecture within the subretinal 
a
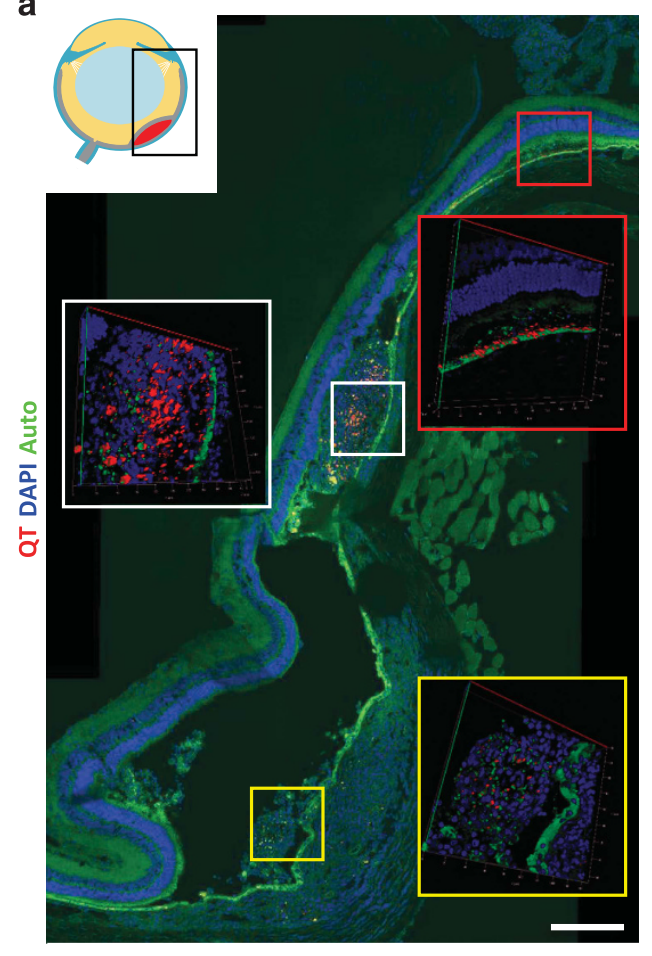

e

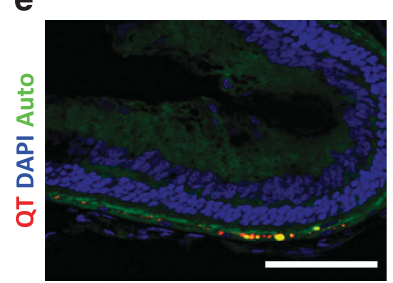

f PPCS + RPEJ

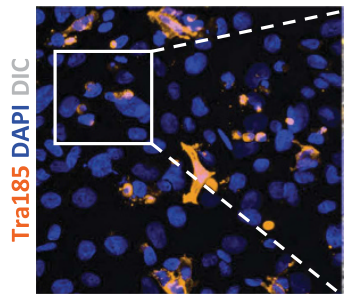

b
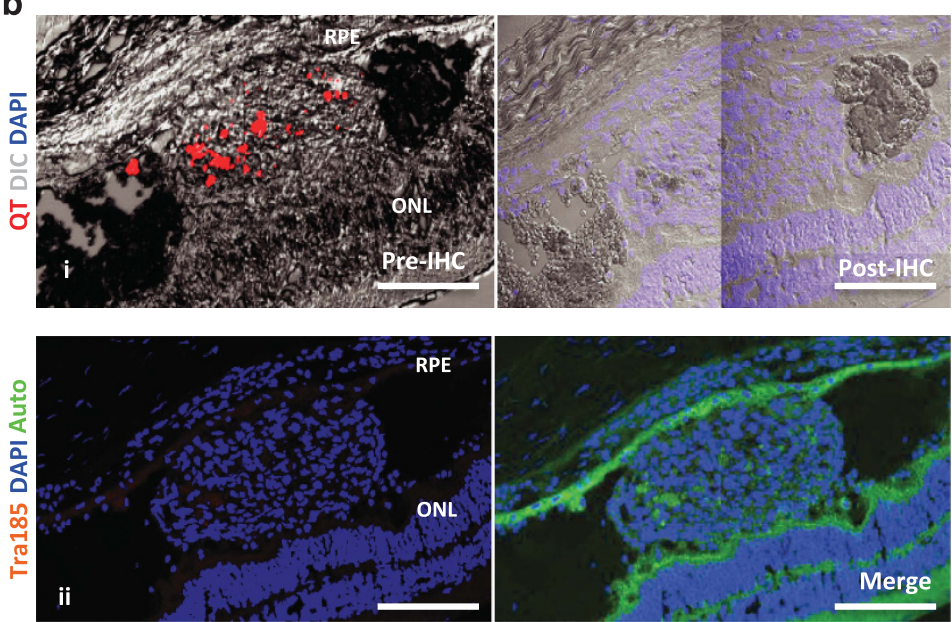

c

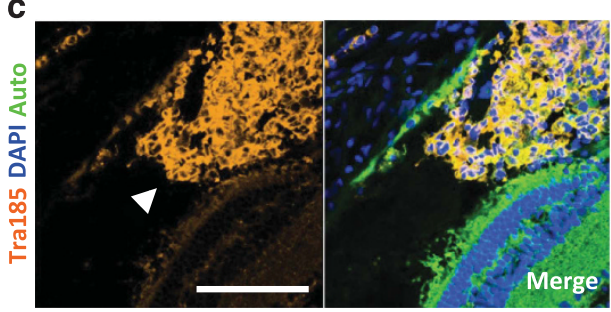

d

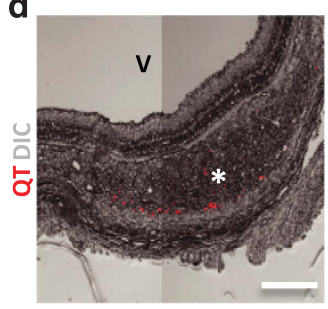

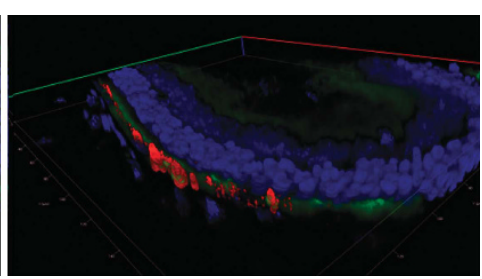

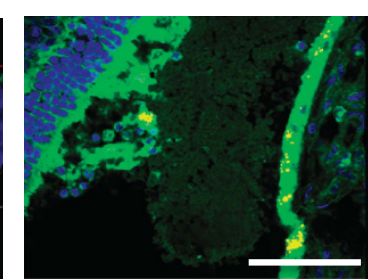

ARPE19 + RPEJ

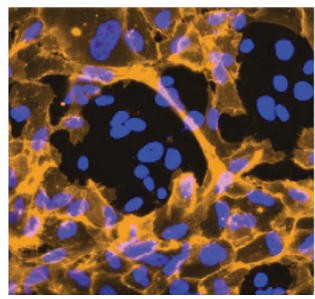



PPCs only

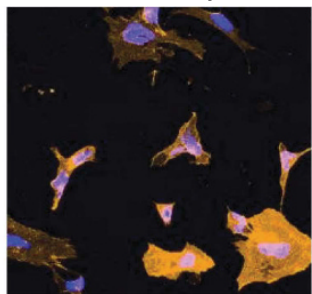

Figure 3 End point Histology of PPC transplants. (a) Representative images of a subretinal bleb containing heterogeneous distribution of Qtracker (QT) graft label at 15 days posttransplant. Nuclei label (DAPI); auto-fluorescence (Auto). (b) (i) Subretinal bleb containing QT exhibiting signal loss after immunohistochemistry (IHC) processing; differential interference contrast (DIC). (ii) Human-specific labeling (Tra185 antibody) did not detect any PPC graft at day 15. (c) Tra185-labeled subretinal PPC-graft (arrowhead) at day 0 posttransplant. (d) Misplaced graft—trace amounts of QT signal in the choroid (asterisk); vitreous (V). (e) Host RPE uptake of QT was found at day 15 posttransplant. (f) Co-cultures of PPCs with rat RPE (RPEJ cell line) showing characteristic phagocytosis of PPCs, while these phagocytosis events were not observed in human (ARPE19 cell line) and rat RPE co-cultures, nor was similar morphology exhibited when cultured alone (PPCs only). (a-e) Scale bars are $250 \mu \mathrm{m}$.

space can also have profound consequences on

engraftment. Such graft characteristics cannot be detected by ophthalmoscopy; however, bimodal imaging provides real-time visualization of subretinal graft thickness and uniformity. Here we show, for the first time, that graft architecture can be highly variable at the time of transplantation, which could represent a considerable source of treatment variation among recipients and across the fundus. In this regard, bimodal imaging allows for early detection of a central problem inherent to cellsuspension therapeutics: undesired graft aggregation resulting in ischemia (reviewed by Robey et $a l^{26}$ ) and/or spatial inhibition of functional connections, such as the highly limited contact observed between bolus PPC-grafts 
and host $\mathrm{RPE}^{9}$ - a common architecture seen here and in other studies $7,9,27)$. Conversely, bimodal imaging can also detect extensive graft dispersion, which can leave insufficient density of cells for functional benefit, as small quantities of integrating PPCs are unlikely to rescue vision. ${ }^{27}$ Adding complexity to graft design is that variation among retinal degenerative diseases and extent of progression are key factors known to greatly affect PPC engraftment. ${ }^{8}$ Given this, requirements for graft architecture will inherently vary among retinal dystrophies and fundus position (eg, in advanced cases where the photoreceptor layer is completely lost or when attempting to rescue the photoreceptor-dense macula). With these factors in mind, bimodal imaging can have an invaluable role in the real-time assessment of novel approaches to generating optimal subretinal graft architecture.

\section{Monitoring biomaterial efficacy in generating optimal graft architecture}

Numerous biomaterials have been used in efforts to optimize subretinal graft architecture..$^{25,28-31}$ However, the performance of these materials cannot be assessed in vivo using standard imaging techniques. A recent (2014) study, implanting a monolayer of RPE attached to a ridged polyester scaffold, utilized a similar approach to our bimodal imaging to help address this very limitation. Using infrared cSLO and OCT, they were able to effectively visualize the scaffold's placement within the subretinal space and fundus position; 32 though, unlike our present study, graft fluorescent-labeling was not used, leaving graft survival to be determined by end point histology. Although this work utilized a disparate cell-therapeutic approach and implantation strategy, it, alongside our study, further illustrates the valuable role in vivo imaging can have in the development of subretinal graft and implant therapeutics.

\section{Study limitations}

Despite superior detection of graft assessment over conventional rodent ophthalmoscopy (summarized in Table 2), certain limitations were observed with bimodal imaging. As QT is a graft-loaded fluorescence, as opposed to expressed fluorescence (eg, GFP transgenic cells), it gives limited evidence of cell viability. Thus QT can only serve as a proxy for graft loss when the signal has decreased, as its persistence does not guarantee live-graft presence. Also, as highlighted in this study, its absence after IHC does not prove graft loss as PPCs are still detectable using human-specific antibodies. Expressed fluorescence, on the other hand, can be used for unambiguous tracking purposes in serial and end point
Table 2 Comparison of bimodal imaging (combined OCT and fCSLO) and ophthalmoscopy for in vivo evaluation of subretinal cell-based transplants in rodents

\begin{tabular}{|c|c|c|c|c|c|c|}
\hline \multirow[t]{2}{*}{ Assessment } & \multicolumn{2}{|c|}{ Ophthalmoscopy } & \multicolumn{2}{|c|}{ OCT } & \multicolumn{2}{|c|}{$f_{c} S L O$} \\
\hline & $d 0$ & Serial & $d 0$ & Serial & $d 0$ & Serial \\
\hline Subretinal graft presence & $\mathrm{i}$ & - & + & $+^{C}$ & $+^{\mathrm{C}}$ & $++^{C}$ \\
\hline Graft fundus position & - & - & + & + & + & + \\
\hline Graft architecture & - & - & + & + & + & + \\
\hline $\begin{array}{l}\text { Microscopic indicators of } \\
\text { host-site inflammation }\end{array}$ & - & - & + & + & - & - \\
\hline Host trauma & + & + & + & + & - & - \\
\hline Graft survival $^{\mathrm{M}}$ & - & - & - & - & + & + \\
\hline
\end{tabular}

Abbreviation: i, implied effectiveness. Effective $(+)$ or ineffective $(-)$ assessment. Effective when data from both OCT and fcSLO are combined (C) if expressed fluorescence is used, as this indicates that live graft is present. Efficacy most evident (M) if expressed fluorescence is used; however, the loss of graft-loaded fluorescence (eg, QT) in the host eye clearly indicates graft loss in that region.

analysis. ${ }^{5,9}$ Utilizing this with bimodal imaging in future studies would allow for high-fidelity, in vivo imaging of graft survival.

The lack of subretinal graft survival we observed at day 15 posttransplant is most likely due to acute rejection, though anoikis and ischemia probably also have a role in diminished graft survival. Evidence of rejection came from bimodal imaging reporting indicators of host-site inflammation at 8 and 15 days posttransplant and that QT signal was found predominantly in the interstitium and/or within host-cell aggregates. Similar aggregates comprised of macrophages and microglia have been observed in subretinal xenograft studies when acute rejection occurred. ${ }^{33}$ Regardless of the cause, lack of graft survival here is not particularly surprising, as PPC survival is considerably low for even syngeneic grafts, ${ }^{9}$ let alone for xenogeneic grafts injected into an inflamed tissue - the dystrophic retina. ${ }^{34,35}$ Further studies will be needed to see whether bimodal imaging can differentiate between viable PPC graft (eg, expressing a far-red fluorophore) and host macrophages/microglia (eg, expressing a disparate cell-specific green fluorophore -MHC-II::EGFP genotype ${ }^{36}$ crossed with a retinal dystrophic mouse). Such application of bimodal imaging would give early real-time insight into the spatiotemporal dynamics of retinal immune responses to PPCs grafts.

\section{Conclusions}

In this study, we have seen advantages in bimodal (OCT-fcSLO) in vivo imaging in preclinical subretinal transplantation trials over conventional imaging. Bimodal imaging enabled serial in vivo detection of cell-suspension graft placement, architecture, indicators of host trauma 
and inflammation, and graft loss. Of particular importance in developing clinically relevant photoreceptor grafts is the information bimodal imaging provides about graft architecture. Taken together, the many capabilities that bimodal imaging offers has the potential to increase efficiency of preclinical trials in cell-based retinal therapeutics.

\section{Summary}

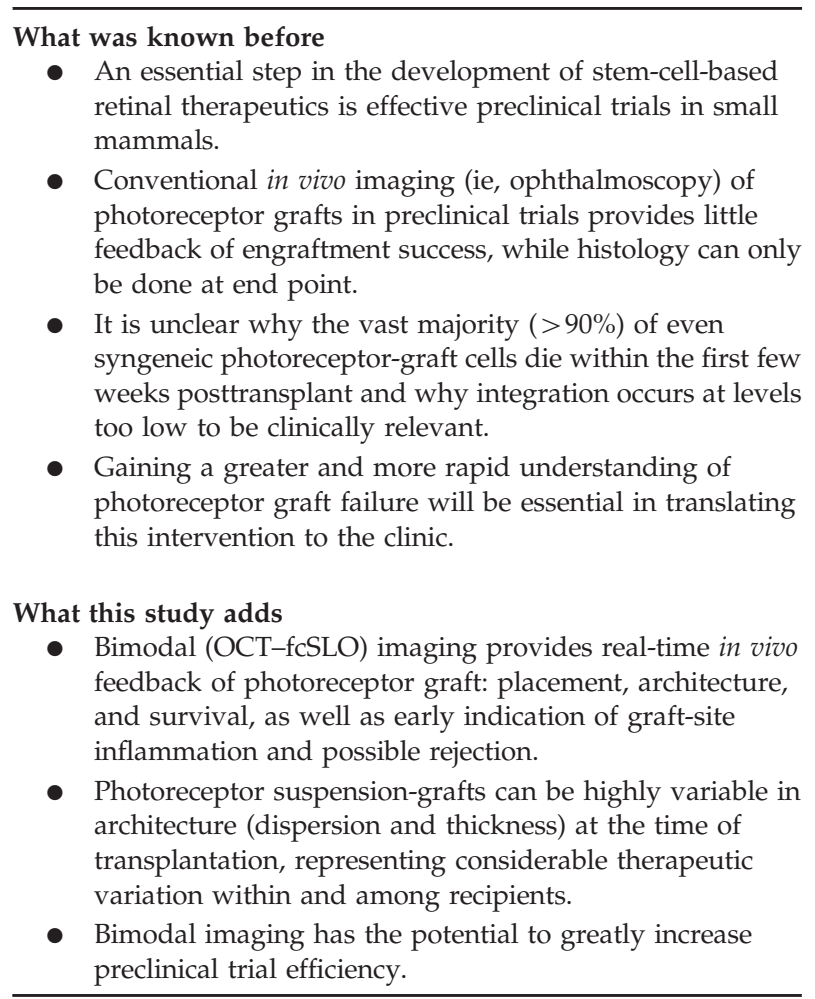

\section{Conflict of interest}

The authors declare no conflict of interest.

\section{Acknowledgements}

This work was supported by: Canadian Institutes of Health Research (CIHR) grants to KGE and MVS, Foundation Fighting Blindness grants to KGE and MVS, Sharon Stewart Trust grant to KGE, and CIHR-CGS-D and University of British Columbia Doctoral grants to CRJL.

\section{Author Contribution}

CRJL: designed and carried out the experiments and data analysis, developed and performed the transplantation procedure described here, assisted with OCT and fcSLO imaging, and wrote the manuscript. ALM: provided critical discussions, perioperative care, and performed OCT and fCSLO imaging. LS: assisted with OCT and fcSLO image processing and gave essential technical assistance. AY: assisted with PPC generation. MVS: provided essential OCT and fCSLO technical assistance and edited the manuscript. KGE: provided essential discussions, experimental design and analysis, edited the manuscript, and supervised the study. All authors read and approved the final manuscript.

\section{References}

1 Pop-Fanea L, Vallespin SN, Hutchison JM, Forrester JV, Seton HC, Foster MA et al. Evaluation of MRI for in vivo monitoring of retinal damage and detachment in experimental ocular inflammation. Magn Reson Med 2005; 53(1): 61-68.

2 Cheng H, Nair G, Walker TA, Kim MK, Pardue MT, Thule PM et al. Structural and functional MRI reveals multiple retinal layers. Proc Natl Acad Sci USA 2006; 103(46): 17525-17530.

3 Qiu G, Seiler MJ, Mui C, Arai S, Aramant RB, de Juan E Jr et al. Photoreceptor differentiation and integration of retinal progenitor cells transplanted into transgenic rats. Exp Eye Res 2005; 80(4): 515-525.

4 Thomas BB, Arai S, Ikai Y, Qiu G, Chen Z, Aramant RB et al. Retinal transplants evaluated by optical coherence tomography in photoreceptor degenerate rats. J Neurosci Methods 2006; 151(2): 186-193.

5 MacLaren RE, Pearson RA, MacNeil A, Douglas RH, Salt TE, Akimoto $\mathrm{M}$ et al. Retinal repair by transplantation of photoreceptor precursors. Nature 2006; 444(7116): 203-207.

6 Warre-Cornish K, Barber AC, Sowden JC, Ali RR, Pearson RA. Migration, integration and maturation of photoreceptor precursors following transplantation in the mouse retina. Stem Cells Dev 2013.

7 Pearson RA, Barber AC, Rizzi M, Hippert C, Xue T, West EL et al. Restoration of vision after transplantation of photoreceptors. Nature 2012; 485(7396): 99-103.

8 Barber AC, Hippert C, Duran Y, West EL, Bainbridge JW, Warre-Cornish $\mathrm{K}$ et al. Repair of the degenerate retina by photoreceptor transplantation. Proc Natl Acad Sci USA 2013; 110(1): 354-359.

9 Singh MS, Charbel Issa P, Butler R, Martin C, Lipinski DM, Sekaran $\mathrm{S}$ et al. Reversal of end-stage retinal degeneration and restoration of visual function by photoreceptor transplantation. Proc Natl Acad Sci USA 2013; 110(3): 1101-1106.

10 Lee D, Geller S, Walsh N, Valter K, Yasumura D, Matthes M et al. Photoreceptor degeneration in Pro23His and S334ter transgenic rats. Adv Exp Med Biol 2003; 533: 297-302.

11 Yanai A, Laver CR, Joe AW, Viringipurampeer IA, Wang X, Gregory-Evans CY et al. Differentiation of human embryonic stem cells using size-controlled embryoid bodies and negative cell selection in the production of photoreceptor precursor cells. Tissue Eng Part C Methods 2013; 19: 755-764.

12 Castanheira P, Torquetti LT, Magalhas DR, Nehemy MB, Goes AM. DAPI diffusion after intravitreal injection of mesenchymal stem cells in the injured retina of rats. Cell Transplant 2009; 18(4): 423-431. 
13 Ballou B, Ernst LA, Andreko S, Harper T, Fitzpatrick JAJ, Waggoner AS et al. Sentinel lymph node imaging using quantum dots in mouse tumor models. Bioconjug Chem 2007; 18(2): 389-396.

14 Pelley JL, Daar AS, Saner MA. State of academic knowledge on toxicity and biological fate of quantum dots. Toxicol Sci 2009; 112(2): 276-296.

15 Summers HD, Brown MR, Holton MD, Tonkin JA, Hondow N, Brown AP et al. Quantification of nanoparticle dose and vesicular inheritance in proliferating cells. ACS Nano 2013; 7(7): 6129-6137.

16 Issaei A, Szczygiel L, Hossein-Javaheri N, Young M, Molday LL, Molday RS et al. Integrated scanning laser ophthalmoscopy and optical coherence tomography for quantitative multimodal imaging of retinal degeneration and autofluorescence. Proc SPIE 2011; 7892: 789209-1-789209-8.

17 Jian Y, Wong K, Sarunic MV. Graphics processing unit accelerated optical coherence tomography processing at megahertz axial scan rate and high resolution video rate volumetric rendering. J Biomed Opt 2013; 18(2): 26002.

18 Nabi IR, Mathews AP, Cohen-Gould L, Gundersen D, Rodriguez-Boulan E. Immortalization of polarized rat retinal pigment epithelium. J Cell Sci 1993; 104(Pt 1): 37-49.

19 Dunn KC, Aotaki-Keen AE, Putkey FR, Hjelmeland LM. ARPE-19, a human retinal pigment epithelial cell line with differentiated properties. Exp Eye Res 1996; 62(2): 155-169.

20 Tzameret A, Sher I, Belkin M, Treves AJ, Meir A, Nagler A et al. Transplantation of human bone marrow mesenchymal stem cells as a thin subretinal layer ameliorates retinal degeneration in a rat model of retinal dystrophy. Exp Eye Res 2013; 118: 135-144.

21 Feng W, Yasumura D, Matthes MT, LaVail MM, Vollrath D. Mertk triggers uptake of photoreceptor outer segments during phagocytosis by cultured retinal pigment epithelial cells. J Biol Chem 2002; 277(19): 17016-17022.

22 Finnemann SC, Bonilha VL, Marmorstein AD, Rodriguez-Boulan E. Phagocytosis of rod outer segments by retinal pigment epithelial cells requires alpha(v)beta5 integrin for binding but not for internalization. Proc Natl Acad Sci USA 1997; 94(24): 12932-12937.

23 Boehm MR, Oellers P, Thanos S. Inflammation and immunology of the vitreoretinal compartment. Inflamm Allergy Drug Targets 2011; 10(4): 283-309.

24 Johnson CJ, Berglin L, Chrenek MA, Redmond TM, Boatright JH, Nickerson JM. Technical brief: subretinal injection and electroporation into adult mouse eyes. Mol Vis 2008; 14: 2211-2226.

25 Diniz B, Thomas P, Thomas B, Ribeiro R, Hu Y, Brant R et al. Subretinal implantation of retinal pigment epithelial cells derived from human embryonic stem cells: improved survival when implanted as a monolayer. Invest Ophthalmol Vis Sci 2013; 54(7): 5087-5096.

26 Robey TE, Saiget MK, Reinecke H, Murry CE. Systems approaches to preventing transplanted cell death in cardiac repair. J Mol Cell Cardiol 2008; 45(4): 567-581.

27 Gonzalez-Cordero A, West EL, Pearson RA, Duran Y, Carvalho LS, Chu CJ et al. Photoreceptor precursors derived from three-dimensional embryonic stem cell cultures integrate and mature within adult degenerate retina. Nat Biotechnol 2013; 31(8): 741-747.

28 Tucker BA, Redenti SM, Jiang C, Swift JS, Klassen HJ, Smith ME et al. The use of progenitor cell/biodegradable MMP2PLGA polymer constructs to enhance cellular integration and retinal repopulation. Biomaterials 2010; 31(1): 9-19.

29 Ballios BG, Cooke MJ, van der Kooy D, Shoichet MS. A hydrogel-based stem cell delivery system to treat retinal degenerative diseases. Biomaterials 2010; 31(9): 2555-2564.

30 Redenti S, Tao S, Yang J, Gu P, Klassen H, Saigal S et al. Retinal tissue engineering using mouse retinal progenitor cells and a novel biodegradable, thin-film poly (e-caprolactone) nanowire scaffold. J Ocular Biol Dis Informatics 2008; 1(1): 19-29.

31 Mazumder MA, Fitzpatrick SD, Muirhead B, Sheardown H. Cell-adhesive thermogelling PNIPAAm/hyaluronic acid cell delivery hydrogels for potential application as minimally invasive retinal therapeutics. J Biomed Mater Res A 2012; 100(7): 1877-1887.

32 Stanzel BV, Liu Z, Somboonthanakij S, Wongsawad W, Brinken R, Eter $\mathrm{N}$ et al. Human RPE stem cells grown into polarized RPE monolayers on a polyester matrix are maintained after grafting into rabbit subretinal space. Stem Cell Rep 2014; 2(1): 64-77.

33 Hambright D, Park KY, Brooks M, McKay R, Swaroop A, Nasonkin IO. Long-term survival and differentiation of retinal neurons derived from human embryonic stem cell lines in un-immunosuppressed mouse retina. Mol Vis 2012; 18: 920-936.

34 Gupta N, Brown KE, Milam AH. Activated microglia in human retinitis pigmentosa, late-onset retinal degeneration, and age-related macular degeneration. Exp Eye Res 2003; 76(4): 463-471.

35 McGill TJ, Prusky GT, Luna G, LaVail MM, Fisher SK, Lewis GP. Optomotor and immunohistochemical changes in the juvenile S334ter rat. Exp Eye Res 2012; 104: 65-73.

36 Boes M, Cerny J, Massol R, Op den Brouw M, Kirchhausen $\mathrm{T}$, Chen $\mathrm{J}$ et al. T-cell engagement of dendritic cells rapidly rearranges MHC class II transport. Nature 2002; 418(6901): 983-988.

Supplementary Information accompanies this paper on Eye website (http://www.nature.com/eye) 